\title{
Kopfschmerz und Migräne - Diagnostische Strategien in der Naturheilpraxis
}

Die Diagnosestellung verschiedener Kopfschmerzarten und der Migräne ist im Grunde genommen relativ einfach und klar: oftmals ergibt sie sich schon aus dem Symptombild und einer guten Schmerzanamnese. Doch besonders wichtig für eine optimale und Erfolg versprechende Therapie ist nicht nur die Diagnose, sondern vor allem das Auffinden der genauen Ursache.

Um eine Grundlage für ein effizientes therapeutisches Vorgehen zu schaffen, ist schon fast das Gespür eines Sherlock Holmes notwendig. Eine beinahe unüberschaubare Menge an Faktoren gilt es zu berücksichtigen. Die nachfolgende Anleitung ist hierfür eine Hilfestellung - wenn auch ohne Anspruch auf Vollständigkeit. Sie zeigt, welche Diagnoseverfahren und Therapieansätze sich in meiner Praxis bewährt haben.

Nach den allgemein üblichen Diagnoseschritten wie Anamnese, Inspektion, Palpation, Perkussion, Auskultation und Funktionsprüfungen sind folgende naturheilkundliche Methoden zur „Ursachenermittlung“ besonders viel versprechend:

- Laborparametrik

- Iridologie

- Dunkelfeldmikroskopie

- Traditionelle Chinesische Medizin

- Das Vertebrale Reaktionssystem (VRS)

- Reflexzonen

und viele andere mehr ...

\section{Klinische Laboruntersuchungen - Kopfschmerzrelevante Para- meter}

Migräne und Kopfschmerzen sind nicht durch Laborparameter ermittelbar, doch können Laboruntersuchungen Ursachen endogener und exogener Art wie Anämie und Polyglobulie (BB, MCH, MCV, MCHC) oder Intoxikationen darstellen. Gerade Belastungen mit Schwermetallen (v. a.
Quecksilber!) und Vergiftungen mit PCB (polychlorierte Biphenyle) können sehr resistente Kopfschmerzen auslösen.

Außerdem sollte laborchemisch eine Infektion ausgeschlossen werden (BB, BSG, CRP, ggf. Antikörpernachweis, Antigene). Oft wird vergessen, dass eine Hyperbilirubinämie migräneartigen Kopfschmerz auslösen kann.

Auch die histaminvermittelten Kopfschmerzen ( siehe auch S. 24) können durch Allergietests und IgE-Erhöhungen im Blut nachgewiesen werden. Doch nicht nur die typischen Laborparameter sollten berücksichtigt werden. Die Untersuchung von Aminosäuren und Vitaminen kann sehr aufschlussreich sein. Oftmals kann hier eine Reduktion von Methionin, Taurin, Vitamin $B_{6}$, Vitamin $B_{1}$ und anderen gefunden werden, die durch Substitution der reduzierten Substanzen (oral oder parenteral) relativ leicht ausgeglichen werden können. Da der Darm häufig einen der wichtigsten Kopfschmerzverursacher im Organismus darstellen kann, sollten dessen Funktionen auf zweierlei Wegen abgeklärt werden: Zum einen hat sich das nutrigene Belastungsprofil zum Ausschluss einer $\mathbf{~ I g G}_{4}$-vermittelten Unverträglichkeit von Nahrungsmitteln (z.B. durch LaborMed, Wiesbaden) gut bewährt. Zum anderen kann die Untersuchung einer Stuhlprobe sehr hilfreich sein (z.B. Labor Enterosan, Bad Bocklet). Hierdurch können nicht nur Dysbiosen, sondern auch der Immunstatus des Darmes sowie mögliche Fehlfunktionen der Darmschleimhaut und der Verdauungsdrüsen (Pankreas, Galle, Leber) ermittelt werden.

\section{Iridologie - Blickpunkt Konsti- tution und Diathese}

Der Iridologie ist es nicht möglich, Kopfschmerzen oder Migräne eindeutig und klar zu diagnostizieren. Ausnahmen hier- von bilden akute Kopfschmerzzustände. Hier kann man vor allem durch die Pupillenreaktionen deutliche Hinweise auf ein zerebrales Schmerzsyndrom erhalten. Existiert z.B. eine einseitige Mydriasis (Weitpupille) mit einer deutlichen Reaktionsarmut, so deutet dies entweder auf ein akutes Schädel-Hirn-Trauma oder auf ein Kopfschmerzsyndrom hin. Eine „Kopfschmerz-Iris“ gibt es nicht.

Doch bietet die Iridologie - was keine andere Diagnosemethode kann - einen direkten Einblick in das Mesenchym eines Menschen. Vor allem genetische Dispositionen und Diathesen, also die Bereitschaft des Organismus an einer bestimmten Stelle (Organ) einzubrechen (Locus minoris resistentiae), kann gut beurteilt werden. Somit gibt es oftmals zahlreiche wertvolle Anzeichen für die mögliche Ursache des Schmerzes. Es ist folglich möglich, die involvierten Systeme des Organismus und seine entsprechende Bereitschaft zu Schmerzen im Gesamten zu ermitteln.

Grundsätzlich kann jeder Mensch zum Kopfschmerz-Patienten werden. Innerhalb der Iridologie kann kein Grundkonstitutionstyp (lymphatisch, hämatogen oder Mischtyp) als besonders schmerzorientiert herausgestellt werden. Erst die Diathesen und Dispositionen geben typische iridologische Hinweiszeichen auf häufig auftretende Kopfschmerzen. Diese sind unter anderem:

- Neurogene Disposition: ein besonders fein strukturiertes Irisstroma mit zahlreichen radiär verlaufenden Fasern (Radiären) kennzeichnet diesen Typus. Der Träger einer neurogenen Disposition spricht in der Regel sehr gut und schnell auf sanftere naturheilkundliche Verfahren an.

- Zirkuläre Furchenbildung als Zeichen einer vegetativ-spastischen Disposition deutet auf genetisch determinierte Probleme im Magnesium-Kalzium-Haushalt 


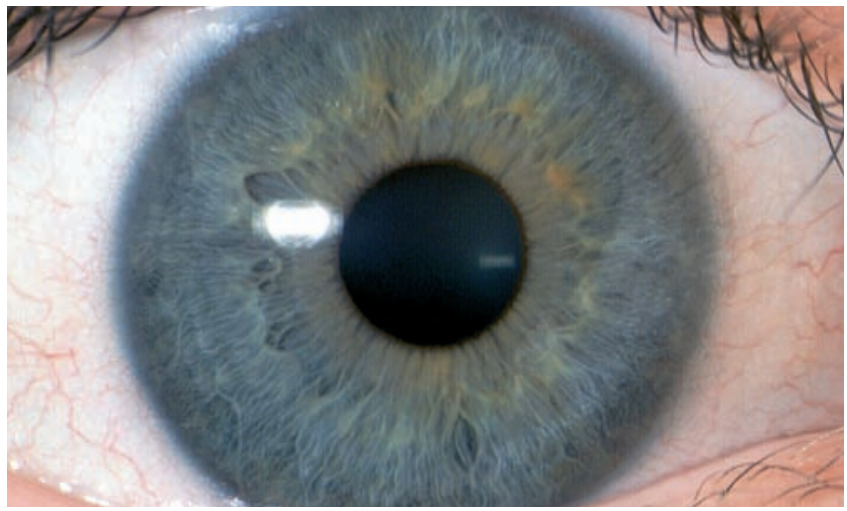

Abb. 1 Rechtes Auge. Lakune mit Reizfaser bei 5 3/4 Uhr spricht für eine genetische Nierenbelastung. Kontrolle der Nierenwerte in Bezug auf die Migräne ist also unerlässlich.

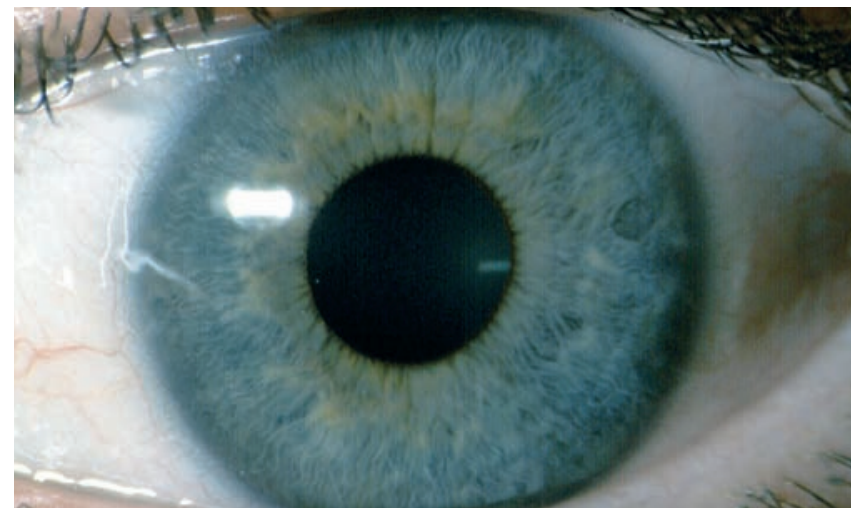

Abb. 2 Linkes Auge einer 32-jährigen Migränepatientin. Lymphatisch-neurogene Konstitution. Pupillenentrundung spricht für Wirbelsäulenprobleme. Pigmentimprägnation von 10 bis 2 Uhr spricht u. U. für zerebrale Stoffwechselstörungen. hin. Zeigt ein Kopfschmerzpatient diese kreisrunden Einziehungen im Ziliarfeld, so sollte die gesamte Therapie mit Magnesium und/oder Kalzium begleitet werden (z.B. als biochemisches Mittel Nr. 7 bzw. Nr. 2 von Nestmann).

- Radiäre Furchenbildung ist ebenfalls ein Anzeichen einer vegetativ-spastischen Disposition: Die vom Pupillenrand in das Ziliarfeld der Iris reichenden Furchen (Einziehungen) deuten häufig auf Durchblutungsstörungen aufgrund von Gefäßverkrampfungen hin. Eine Gefäß entspannende Therapie bringt bei Migräne Patienten oft Erleichterung. Vitamin-E-Präparate (z.B. Vitamin-E-400mg, Wörwag) tragen zur Gefäßentspannung bei. Auch Secale-cornutum- (z.B. Secale corn. Spl ${ }^{\circledR}$, Pascoe) oder Rutinum-Präparate (z.B. Rutinum Synergon Nr. 3b ${ }^{\circledR}$, Kattwiga) sollten hier eingesetzt werden.

- Pigmentationen, z.B. gelbliche bis grünlich-gelbliche Einlagerungen in oder auf das Irisstroma (10-2 Uhr), deuten häufig auf Störungen im zerebralen Proteinstoffwechsel hin (@ Abb 2). Aminosäuren-Präparate (z.B. Cerebro Kps. ${ }^{\circledR}$, Landhaus-Naturprodukte) zusammen mit einem Stoffwechselmittel (Cicuta virosa Synergon Nr. 124 ${ }^{\circledR}$, Kattwiga) können hilfreich sein.

" „Organzeichen“: Zeigen sich Veränderungen im Irisstroma oder Pigmenteinlagerungen in bestimmten Bereichen, so kann häufig ein Bezug zwischen diesem Organ und der Kopfschmerzsituation hergestellt werden. Eine Behandlung mit entsprechenden organbezogenen Arzneien ist dann immer notwendig (๑ Abb 1).
Lebertee bei Leberbelastung nach Josef Karl bestehend aus:

Fol. Boldo 20.0, Rad. C. Hb. Taraxaci 50.0, Rhiz. Curcumae 15.0, Fol. Menth. Pip. 15.0, Rad. Chichorii 15.0, Flor. Lavendulae 10.0

Nierenmittel bei Nierenzeichen: z. B. Nierentonikum Nestmann

Zusammen mit den konstitutionellen Hinweisen ist es so möglich, entweder eine weiterführende Diagnostik anzustreben oder sofort, gewissermaßen „aus dem Auge heraus" einen therapeutisch Erfolg versprechenden Weg einzuschlagen.

\section{Dunkelfeldmikroskopie - Der Ist- Zustand wird im Dunkelfeld erhellt}

So wie die Iridologie Hinweise auf die konstitutionellen Faktoren des Menschen ermöglicht, so gibt das Dunkelfeldbild direkte Auskunft über seinen Ist-Zustand. Auch durch die dunkelfeldmikroskopische Untersuchung ist es nicht möglich, einem Patienten sofort „auf den Kopf hin“ zu sagen, ob er unter Kopfschmerzen leidet oder nicht. Doch auch durch dieses Verfahren kann man die möglichen Ursachen weiter „einkreisen“.

Neben möglichen bakteriellen und mykotischen Belastungen zeigen sich vor allem Durchblutungsstörungen und subazide Zustände im Dunkelfeldbild sehr früh. Besonders deutlich werden diese
z.B. durch die so genannte „Geldrollen“Bildung dargestellt. Auch Filitbildungen und Fibrinnetze deuten auf eine Durchblutungserschwernis hin.

Eine Therapie mit entsprechenden, den Säure-Basen-Haushalt regulierenden Präparaten ist hier häufig notwendig (z.B. Baso-Syx Syxy $\left.{ }^{\circledR}\right)$. Isopathische Therapeutika (Sanum-Präparate) sollten entsprechend des Dunkelfeldbildes gewählt werden. Oftmals zeigen sich Präparate wie das Mucokehl ${ }^{\circledR}$ (Sanum), das Latensin ${ }^{\circledR}$ (Sanum) oder das Quentakehl ${ }^{\circledR}$ (Sanum) als wirkungsversprechend.

Die Dunkelfelduntersuchung unterstützt außerdem die Störfelddiagnostik (siehe unten).

\section{Sto̊rfelddiagnostik - Was sto̊rt den Therapieerfolg?}

Frustrierend für Patient und Behandler ist es, wenn die bewährtesten Therapien ohne Erfolg bleiben. Besteht eine solche Therapieresistenz in der Anamnese oder im Behandlungsverlauf, so sind häufig ein Störfeld oder ein Herdgeschehen schuld daran. Zudem können Störfelder selbst kopfschmerz- oder migräneauslösend sein!

Die Störfeldsuche kann sich leider mitunter relativ schwierig gestalten. Es sollten alle Narben auf Empfindungsveränderungen überprüft werden. Finden sich hier dysästhetische Narben-Kolloide, so kann durch die intrakutane Injektion von Procain $1 \%$ (z.B. von Loges) eine so genannte Narbenentstörung erreicht werden 


\section{Im Dunkeln nach dem Störfeld suchen}

In der dunkelfeldmikroskopischen Untersuchung zeigen sich bei Störfeldbelastungen häufig so genannte Nuggets (goldgelblich schimmernde Proteinansammlungen). Auch Anzeichen von Leptotrichia buccalis können auf einen Herd hindeuten. Sind diese vorhanden, ist in der Regel ein bakterieller Herd die Ursache und muss entsprechend gesucht werden. Innere Herde und Störfelder jedoch können meist nur durch Provokation ermittelt werden. Eine Möglichkeit zur provokativen Aktivierung stellen z. B. die Spenglersan Kolloide D oder Dx ${ }^{\circledR}$ (Meckel) dar. Auch die intramuskuläre Injektion von 5-7 ml nativem Eigenblut oder die Gabe von Utilin ${ }^{\circledR}$ S (säurefest, Sanum) können zu einer Herdprovokation führen.

Cave: Wird eine solche Provokation durchgeführt, muss unter Umständen mit sehr starken Reaktionen des Patienten gerechnet werden. Dies kann neben Fieber, Zahnschmerzen oder einer aktivierten Sinusitis durchaus auch eine akute Appendizitis bedeuten. Eine gute Aufklärung (mit Dokumentation) und besondere Sorgfalt während Diagnose, Behandlung und Überwachung des Patienten sind also absolut notwendig!

Zahnherde können oft gut durch die Elektroakupunktur nach Voll aufgefunden werden. Diese müssen jedoch von einem Zahnmediziner saniert werden.

\section{Traditionelle Chinesische Medizin - Vier Meridiane und vier Kopfschmerzarten (OS.40)}

Die TCM bietet ein komplettes System zum Auffinden und Beseitigen von Kopfschmerzen und Migräne an. Grob können vier Migränearten eingeteilt werden:

- Blasenmigräne: Schmerzbeginn im nasalen Orbitalwinkel, meist mit der gesamten Wirbelsäule in Kontakt stehend,

- Nierenmigräne: Schmerzbeginn hinter den Augen, meist mit den Ausleitungssystemen verknüpft,

- Gallenmigräne: Schmerzbeginn im Nacken, meist mit der Leber-Galle-Funktion verbunden,

- Magenmigräne: Schmerzbeginn im Stirnbereich, meist mit gastrointestinalen Symptomen.

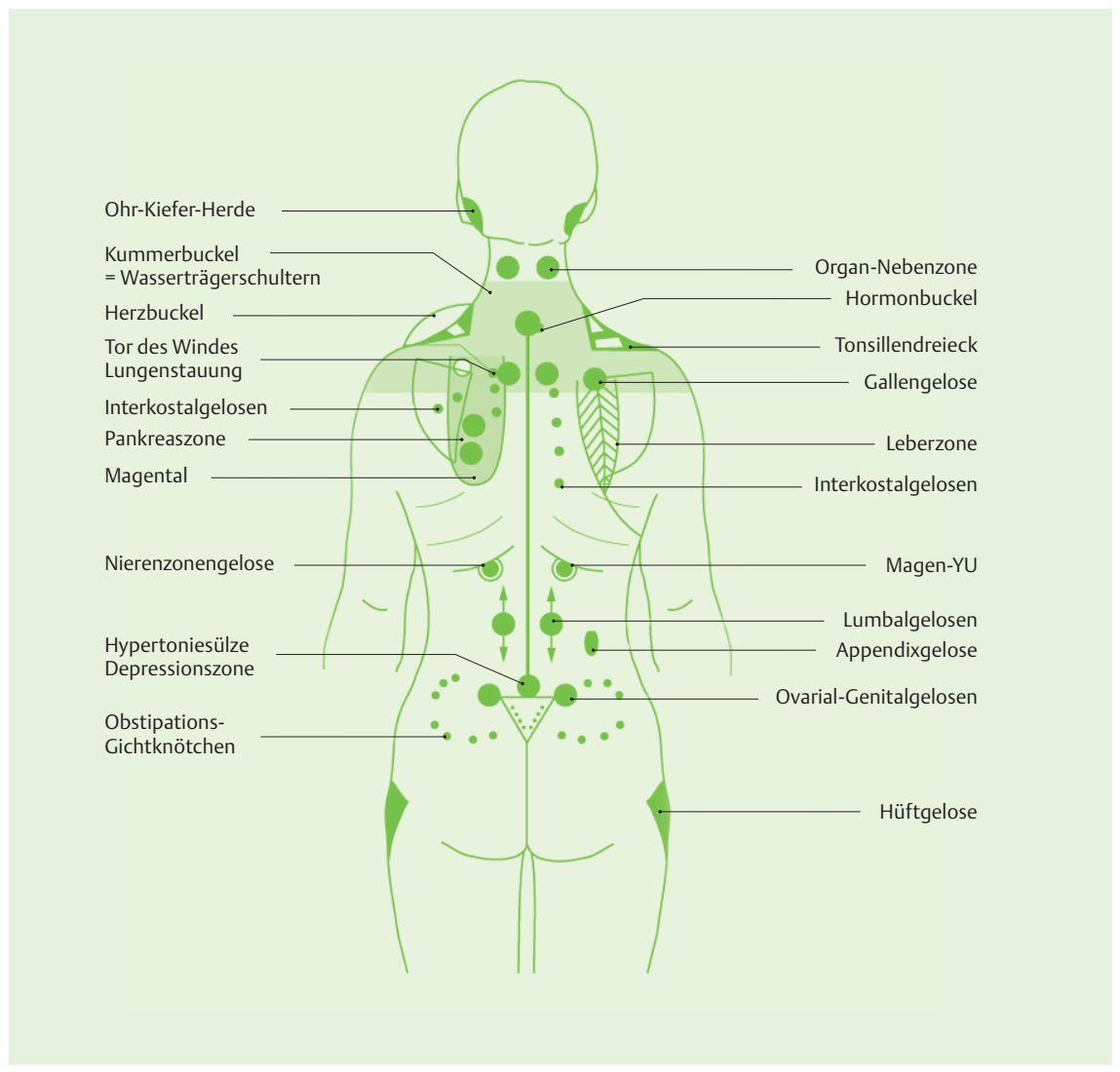

Abb. 3 Schröpfzonen nach Abele.

\section{Das Vertebrale Reaktionssystem (VRS) - vernetzt über die Wirbel- såule}

Das Vertebrale Reaktionssystem (VRS) setzt sich aus allen mit der Wirbelsäule verbundenen Strukturen zusammen, also aus Knochen, Muskeln, Sehnen, Bändern, Blutgefäßen, Rückenmark etc. Nahezu jede Reaktion des Organismus zeigt sich früher oder später in einer Veränderung eines dieser Anteile des VRS. Fast alle Kopfschmerz- und Migräne-Patienten weisen eine Blockade der HWS, vornehmlich des Atlas-Axis-Gelenkes, auf.

Doch die gesamte Gangkette (vom Fußgelenk bis zum Atlas) kann gestört sein. Blockaden einzelner Wirbel, der Rippengelenke oder des Iliosakralgelenks führen ebenfalls häufig zu Kopfschmerz und Migräne. Auch das Kiefergelenk darf nicht vergessen werden! Verspannungen der Mm. masseter oder Fehlbisse (z.B. Zahnfrühkontakte) können zu massiven Verspannungen der HWS führen und somit migränetriggernd funktionieren ( siehe auch S. 22). Das VRS reagiert jedoch auch auf viszerale Reize. Sind innerorganische Störungen vorhanden, wird das VRS ständig mitgereizt, was zu Myogelosen oder Blockaden im entsprechenden Segment führen kann (Segmentdiagnostik nach Huneke).

Als therapeutische Konsequenz erfolgt hier entweder eine sanfte Mobilisationstherapie (z.B. Wirbeltherapie nach Dorn oder Osteopathie) oder Massagen. Auch paravertebrale Injektionsserien, z.B. mit Procain $1 \%$ i.c. + Spondylose-Injektopas ${ }^{\circledast}$ (Pascoe) können hilfreich sein.

\section{Reflexzonen als Verräter der Ursachen}

Bereits einfache Untersuchungen der bekannten Fuß-, Ohr-, Gesichts- oder Rückenreflexzonen können die Diagnostik der möglichen Ursachen mit wenig Aufwand weiter präzisieren.

Besonders effektiv ist häufig die Untersuchung der Schröpfzonen nach Abele. Schon bei einer kurzen Inspektion des Rückens können Myogelosen und Hautveränderungen (auch Pigmentationen) auffallen. Zahlreiche Nävi (Leberflecken) z.B. zeigen die Beteiligung von Leber und/oder Galle mit an. Myogelosen im Bereich der Schulterblätter geben, wenn sie linkssei- 
tig auftreten, einen Hinweis auf eine Pankreas-Beteiligung und rechtsseitig wiederum auf die Leber. Eine „gequollene“ Region rings um den Prominens (C 7) deutet meist auf einen hormonellen Einfluss auf die Kopfschmerz- oder Migräne-Situation hin.

\section{Es gibt so viele Mo̊glichkeiten ...}

... die tatsächlichen Ursachen von Migräne und Kopfschmerzen zu ermitteln. Das Wichtigste überhaupt bei der Ursachensuche ist, nicht den Mut zu verlieren und geduldig eine Möglichkeit nach der anderen auszuschöpfen. Manchmal jedoch kann auch eine Ausschlusstherapie Erfolg versprechend sein. Vor allem dann, wenn der Patient sich weitere (teure) Untersuchungen nicht leisten kann. Bei einer Ausschlusstherapie wird der Verdacht therapiert. Kommt z.B. eine Intoxikation als Verursacher für die Kopfschmerzen infrage und ist eine Untersuchung auf alle potenziellen Toxine für den Patienten nicht durchführbar, so kann durch die Gabe einer so genannten „Entgiftungskombination “ [Leber-, Nieren- und Lymphmittel, z.B. Quassia ${ }^{\circledR}$ Spl (Pascoe), Juniperus ${ }^{\circledR}$ Spl
(Pascoe) und Lymphdiaral ${ }^{\circledR}$ (Pascoe)] die Entgiftung einfach angeregt werden. Hat die Therapie Erfolg, so lag man mit dem Verdacht richtig. Hat sie keinen Erfolg, so muss nach anderen Ursachen gesucht werden. Es könnte auch eine Therapieblockade vorliegen, die vorher beseitigt werden muss.

\section{(1) Weitere Literaturtipps}

Abele U: Die Aschner-Fibel. 13. Aufl. Stuttgart: Haug; 1996 (Ausleitungsverfahren)

Bleker M: Blutuntersuchung im Dunkelfeld. Hoya: Semmelweis-Institut; 1997 (Dunkelfeldmikroskopie)

Hauser W, Stolz R, Karl J: Informationen aus Struktur und Farbe. 2. Aufl. Gerlingen: Felke Institut; 2004 (Iridologie)

Jahn C: Herdbelastungen und Störfelder der Kopfregion. 1. Aufl. Beuren: Grauer Verlag; 2001 (Störfelddiagnostik)

Stux G.: Akupunktur. Lehrbuch und Atlas. 6. Aufl. Heidelberg/Berlin: Springer; 2003

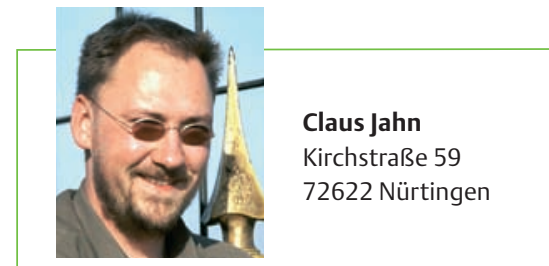

HP Claus Jahn ist seit 9 Jahren in eigener Naturheilpraxis niedergelassen. Er arbeitet als freier Mitarbeiter in einem iridologischen Lehr- und Forschungsinstitut, dem Felke-Institut (Gerlingen). Neben seinen Praxis- und seinen Vortragstätigkeiten hat er zahlreiche Veröffentlichungen verfasst; unter anderem ein Buch zur Kopfschmerzund Migränediagnostik.

E-Mail: c.jahn@naturheilpraxis-jahn.de 